\title{
A Digital Platform for Local Foodborne IIIness and Outbreak Surveillance
}

\author{
Jared B. Hawkins ${ }^{* 1}$, Gaurav Tuli ${ }^{1}$, Sheryl Kluberg ${ }^{1,4}$, Jenine Harris ${ }^{2}$, John S. \\ Brownstein $^{1,3}$ and Elaine Nsoesie ${ }^{2}$
}

'Boston Children's Hospital, Boston, MA, USA; ${ }^{2}$ Washington University in St. Louis, St. Louis, MO, USA; ${ }^{3}$ Harvard Medical School, Boston, MA, USA; ${ }^{4}$ Boston University, Boston, MA, USA

\section{Objective}

Develop a platform to enable local surveillance of foodborne illness reported on social media and restaurant review sites for supplementing traditional foodborne disease surveillance programs. In this presentation, we will discuss our collaboration with local public health departments to develop a foodborne disease surveillance Dashboard.

\section{Introduction}

Foodborne illness affects 1 in 4 Americans, annually. However, only a fraction of affected individuals seek medical attention. To supplement traditional approaches to foodborne disease surveillance, researchers and public health departments are considering reports of foodborne illness on social media sites $[1,2]$. In this project, we work with local public health departments to develop a platform that uses digital data sources such as, Twitter and Yelp, to supplement foodborne disease surveillance efforts. In addition to monitoring reports of illness, this platform can also be used to respond to suspected foodborne illness reports and spur restaurant inspections to ensure food safety. To this end, we have developed a Dashboard that monitors social media chatter for reports of food poisoning in real-time. The Dashboard facilitates responding to illness reports and contacting consumers to provide additional information through a reporting form. The Dashboard is low cost, easy to use and designed to enable easy implementation for any region.

\section{Methods}

Our database currently consist of 1.5 million foodservice reviews and 680 million tweets. For the tweets, approximately $10 \%$ have a geo-coordinate provided by the users. We inferred the geocoordinates of another $46 \%$ of tweets using the 'location' field from the Twitter user profile by querying the Google Maps API. For automated detection of foodborne illness reports, we first develop a list of keywords consisting of foodborne disease symptoms and disease names. Next, we use text matching to filter the reports that contain at least one of the keywords. We then use a supervised machine learning classifier to extract the relevant reports. A report, for example, in which an individual mentions experiencing food poisoning after eating at a restaurant is considered relevant. However, a report is considered irrelevant when a keyword is used in another context (e.g. "this restaurant is sick!"). We developed a support vector machine classifier (SVM) that aims to create maximum separation between the irrelevant and relevant reports by identifying the optimal hyperplane. The process of developing a reliable classifier is iterative and requires refinement over multiple rounds of feature selection and parameter configuration.

\section{Results}

The SVM classifier was evaluated using 6084 tweets. The classifier had an accuracy and precision of $85 \%$ and $82 \%$, respectively. These performance results are promising, especially since the training set was unbalanced and relevant and irrelevant tweet classes were extremely similar. We next ran the classifier on a real-time Twitter stream of tweets containing at least one foodborne illness keyword. Over a four-month period in 2015, approximately $50 \%$ of the tweets were identified by the classifier as being true self-reported food poisoning incidents (Figure 1).

\section{Conclusions}

Restaurants with lower food safety scores have been associated with higher outbreak reports [3]. Real-time surveillance of foodborne illness reports can aid local public health departments to identify and limit the spread of foodborne disease outbreaks.

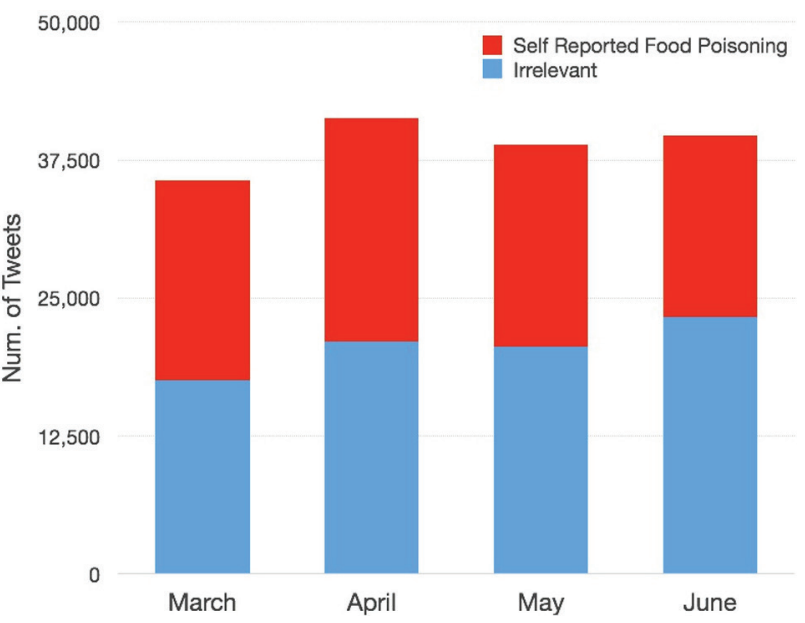

Keywords

Foodborne illness; Surveillance; Social media; Outbreak

\section{References}

1. Nsoesie EO, et al. Online reports of foodborne illness capture foods implicated in official foodborne outbreak reports. Prev Med. 2014;67:264-9.

2. Harris JK, et al. Health Department Use of Social Media to Identify Foodborne Illness - Chicago, Illinois, 2013-2014. Morbidity and Mortality Weekly Report. 2014;63(32):681-5.

3. Irwin $\mathrm{K}$, et al. Results of routine restaurant inspections can predict outbreaks of foodborne illness: the Seattle-King County experience. Am J Public Health. 1989;79(5):586-90.

\section{*Jared B. Hawkins}

E-mail: jared.hawkins@childrens.harvard.edu 